\section{Response to Cody}

In response to our commentary on the classification of lowpenetrance alleles, ${ }^{1}$ Cody presents a clinically derived alternative to current ClinVar classifications that aims to address the growing body of evidence for complex variant effects and to accommodate difficult-to-interpret, lowpenetrance alleles. One of the primary arguments presented for this revised system is the goal of creating a system that puts clinical utility at the forefront and prioritizes the patient's treating physician as the end user. Building on this concept with a focus on probability as the metric for variant classification over the traditional benign versus pathogenic dichotomy, we would like to further extend this discussion to include quantitative models of variant interpretation and how they can be implemented by clinicians to guide patient care.

While the system suggested by Cody increases the granularity of current ClinVar classifications, we believe that fully embracing the probabilistic nature of variant interpretation to adopt a quantitative model would better address the identified weaknesses of the current system. To this end, we have found the widely used American College of Medical Genetics and Genomics/Association for Molecular Pathology (ACMG/AMP) variant classification guidelines ${ }^{2}$ to be a more conducive framework. Tavtigian et al. demonstrated that although they were designed as a qualitative set of criteria, the logic used in the ACMG/AMP guidelines is mathematically founded and compatible with Bayesian statistical reasoning. It was shown that the ACMG/AMP guidelines could be modeled as a naïve Bayesian classifier taking the qualitative evidence categories as inputs and outputting a quantitative probability of pathogenicity for individual variants. ${ }^{3}$

Formally, the output of the Bayesian classifier (designated "post_P") represents the probability of pathogenicity for an individual variant given the provided evidence. Functionally, interpretation of this probability is similar to the current categorical classifications ranging from benign to pathogenic. ${ }^{3}$ More importantly, however, are the opportunities enabled by having a quantifiable metric. A major limitation of the current ACMG/AMP guidelines is that variants are assessed individually. In clinical practice, the patient's entire genetic profile must be considered as a whole and the effect of individual variants cannot be assessed in isolation. Variants in the same gene must be considered in combination since the pathogenicity of a specific variant in a given patient is dependent upon that patient's other variants. Having a quantitative measure of probability allows us to address this limitation by evaluating probabilities together. Mathematically, multiplying the pathogenic probabilities of two variants together represents the probability of both variants being pathogenic and in the context of Mendelian genetics (assuming recessive inheritance) also represents the probability that the two variants are phenotypically relevant. The effects of two pathogenic variants in combination is ultimately what clinicians and the patient are most interested in and the significance of the finding can be further validated by matching the identified gene's function to the patient's clinical presentation.

To systematically evaluate the combined variant effects across a patient's sequencing results, we envision a system where the probabilities of the two variants with the highest probability of pathogenicity in each gene are multiplied together to generate a probability score for each gene. These scores can be ranked or filtered based on predetermined thresholds with the option for genes matching the patient's clinical presentation to have lower thresholds. This would result in a short list of candidate genes containing genes with a high probability of two pathogenic variants or a moderate probability of two pathogenic variants for genes matching clinical presentation. From a clinician's perspective, this dramatic reduction in number of candidate variants not only streamlines the process but also minimizes room for bias or error.

Extending this concept beyond Mendelian genetics brings up the discussion of low-penetrance alleles and the varying thresholds of mutation burden for different conditions. As highlighted in our previous commentary, the role of hypomorphic alleles is well-documented. However, where they fit into current variant classification systems is much less clear. ${ }^{1}$ The concepts of mutational severity (the degree to which a variant negatively affects protein structure and function) and probability of pathogenicity (the degree to which we understand that a variant contributes to disease cause) are often closely intertwined and difficult to separate. The distinction between these two measures is important in the context of hypomorphic alleles because a variant with high probability of a mild effect is different than a variant with uncertain probability of a stronger effect. In these cases, interpretation will depend heavily on the context of the variant and rely on information external to the classification of the variants themselves. Clinical information such as severity of clinical presentation, age of onset, family history, and nature of the condition can be used to qualitatively assess the meaning of the probability scores derived from the Bayesian model. Furthermore, this information can be used to adjust the thresholds for combined variant effects between genes or patients. Conditions known to have a broad spectrum of clinical presentation may prompt lower thresholds for relevant genes and be more inclusive of variants with lower pathogenicity scores whereas conditions that are more binary in their presentation may rely on stricter thresholds. Similarly, patients with milder presentation or later age of 
onset may warrant reduced thresholds to increase consideration for hypomorphic alleles.

This flexibility in interpretation could facilitate the inclusion of the cases detailed in our previous commentary ${ }^{1}$ and identified by Cody into existing variant classification models and extend the utility of the ACMG/AMP guidelines. While substantial efforts will be required to formulate such a system and validate its applicability across the broad spectrum of clinical genetics, we believe that this would be a significant step forward in standardizing variant interpretation. The transition from a single variant, qualitative classification system to a multivariant, quantitative system would better suit the needs of clinicians while creating a framework for the systematic interpretation of sequencing results. The flexibility of a quantitative, probabilistic model also provides significant opportunities for modified implementations to be adapted to different disease mechanisms.

\section{DISCLOSURE}

The authors declare no conflicts of interest.

Publisher's note: Springer Nature remains neutral with regard to jurisdictional claims in published maps and institutional affiliations.

Nicholas K. Wang, MHS ${ }^{1}$ and

John P. W. Chiang, PhD, FACMG (D) ${ }^{1}$

${ }^{1}$ Molecular Vision Laboratory, Hillsboro, OR, USA. Correspondence:

John P. W. Chiang (jchiang@mvisionlab.com)

\section{REFERENCES}

1. Wang NK, Chiang JPW. Increasing evidence of combinatory variant effects calls for revised classification of low-penetrance alleles. Genet Med. 2019;21:1280-1282.

2. Richards S, Aziz N, Bale $S$, et al. Standards and guidelines for the interpretation of sequence variants: a joint consensus recommendation of the American College of Medical Genetics and Genomics and the Association for Molecular Pathology. Genet Med. 2015;17:405-424.

3. Tavtigian SV, Greenblatt MS, Harrison SM, et al. Modeling the ACMG/ AMP variant classification guidelines as a Bayesian classification framework. Genet Med. 2018;20:1054-1060.

(1) (2) Open Access This article is licensed under a Creative Commons cc) ${ }_{\mathrm{BY}} \mathrm{NC}_{\mathrm{SA}}$ Attribution-NonCommercial-ShareAlike 4.0 International License, which permits any non-commercial use, sharing, adaptation, distribution and reproduction in any medium or format, as long as you give appropriate credit to the original author(s) and the source, provide a link to the Creative Commons license, and indicate if changes were made. If you remix, transform, or build upon this article or a part thereof, you must distribute your contributions under the same license as the original. The images or other third party material in this article are included in the article's Creative Commons license, unless indicated otherwise in a credit line to the material. If material is not included in the article's Creative Commons license and your intended use is not permitted by statutory regulation or exceeds the permitted use, you will need to obtain permission directly from the copyright holder. To view a copy of this license, visit http://creativecommons.org/licenses/by-nc-sa/4.0/.

(C) The Author(s) 2019

Advance online publication 9 August 2019. doi:10.1038/s41436-019-0621-Z 